\title{
Measurement properties of painDETECT by average pain severity
}

This article was published in the following Dove Press journal:

ClinicoEconomics and Outcomes Research

10 November 2014

Number of times this article has been viewed

Joseph C Cappelleri'

E Jay Bienen²

Vijaya Koduru ${ }^{3}$

Alesia Sadosky ${ }^{4}$

'Pfizer, Groton, CT, ${ }^{2}$ Outcomes research consultant, New York, NY,

${ }^{3}$ Eliassen Group, New London,

CT, USA; ${ }^{4}$ Pfizer, New York, NY, USA
Background: Since the burden of neuropathic pain $(\mathrm{NeP})$ increases with pain severity, it is important to characterize and quantify pain severity when identifying $\mathrm{NeP}$ patients. This study evaluated whether painDETECT, a screening questionnaire to identify patients with NeP, can distinguish pain severity.

Materials and methods: Subjects ( $n=614,55.4 \%$ male, $71.8 \%$ white, mean age 55.5 years) with confirmed NeP were identified during office visits to US community-based physicians. The Brief Pain Inventory - Short Form stratified subjects by mild (score $0-3, n=110$ ), moderate (score 4-6, n=297), and severe (score 7-10, n=207) average pain. Scores on the nine-item painDETECT (seven pain-symptom items, one pain-course item, one pain-irradiation item) range from -1 to 38 (worst $\mathrm{NeP}$ ); the seven-item painDETECT scores (only pain symptoms) range from 0 to 35 . The ability of painDETECT to discriminate average pain-severity levels, based on the average pain item from the Brief Pain Inventory - Short Form (0-10 scale), was evaluated using analysis of variance or covariance models to obtain unadjusted and adjusted (age, sex, race, ethnicity, time since NeP diagnosis, number of comorbidities) mean painDETECT scores. Cumulative distribution functions on painDETECT scores by average pain severity were compared (Kolmogorov-Smirnov test). Cronbach's alpha assessed internal consistency reliability.

Results: Unadjusted mean scores were 15.2 for mild, 19.8 for moderate, and 24.0 for severe pain for the nine items, and 14.3, 18.6, and 22.7, respectively, for the seven items. Adjusted nineitem mean scores for mild, moderate, and severe pain were 17.3, 21.3, and 25.3, respectively; adjusted seven-item mean scores were 16.4, 20.1, and 24.0, respectively. All pair-wise comparisons of scores between pain-severity groups showed sizable and statistically significant differences $(P<0.0001)$. Cumulative distribution functions showed distinct separation between severity $(P<0.0001)$. Cronbach's alphas were 0.76 and 0.80 for the nine- and seven-item scales, respectively.

Conclusion: This study provides strong psychometric evidence on the validity and reliability of painDETECT for distinguishing average pain severity in patients with $\mathrm{NeP}$.

Keywords: neuropathic pain, painDETECT, pain severity, psychometric properties

\section{Introduction}

Neuropathic pain (NeP), a common cause of chronic pain, results from a variety of diseases and medical conditions, and is defined as pain caused by a lesion or disease of the peripheral somatosensory nervous system. ${ }^{1}$ Although heterogeneity in epidemiologic studies has been a limitation in estimating its presence in the population, best estimates suggest that the overall prevalence of pain with neuropathic characteristics is between $7 \%$ and $10 \%{ }^{2}$ However, when $\mathrm{NeP}$ is present, it is associated with a substantial 
socioeconomic burden that has been characterized not only for conditions that are well recognized (eg, painful diabetic peripheral neuropathy and chronic low-back pain) but also for several that are less frequent (eg, small-fiber neuropathy). ${ }^{3-7}$ Furthermore, evidence suggests that the patient and economic burdens are higher at greater pain-severity levels. ${ }^{3-7}$

Because there is only weak evidence for interventional management of $\mathrm{NeP},{ }^{8}$ treatment relies on a pharmacologic approach, and guidelines for pharmacologic management of NeP have been published. ${ }^{9,10}$ Despite these recommendations and the availability of medications from several drug classes, NeP management remains challenging, not least because of the need for appropriate identification of NeP when making treatment decisions.

To identify the presence of $\mathrm{NeP}$ in patients reporting chronic pain, differences in the symptoms and descriptions of $\mathrm{NeP}$ relative to nociceptive pain were investigated to develop various instruments to screen for the likelihood that pain is of neuropathic rather than nociceptive origin. ${ }^{11}$ While some instruments, such as the DN4 (Douleur Neuropathique 4) ${ }^{12}$ and the original Leeds Assessment of Neuropathic Symptoms and Signs (LANSS) ${ }^{13}$ include a requirement for clinical examination, other instruments, such as a patient self-report version of the LANSS (S-LANSS), ${ }^{14}$ as well as painDETECT, ${ }^{15}$ do not require clinical examination. In particular, painDETECT is a simple, fully patient-reported questionnaire that has been validated for screening and identification of components of NeP. ${ }^{15}$ In contrast to S-LANSS, which evaluates sensory symptoms based on a dichotomous response (yes/no), painDETECT consists of 6-point Likert-type scales, which provide a broader range or continuum of assessment. Since pain severity is not only of immediate importance to patients but also increases the overall burden, characterizing pain-severity levels when identifying patients with $\mathrm{NeP}$ can help inform treatment decisions. Although an association between pain severity and painDETECT scores was suggested in several studies, including the original painDETECT-validation study, ${ }^{15-17}$ this relationship has not been psychometrically evaluated with the goal of differentiating NeP severity. Therefore, the purpose of this study was to determine whether painDETECT can differentiate among levels of average pain severity in patients with NeP.

\section{Materials and methods Data source}

Data for this analysis were obtained during a cross-sectional, observational study on the burden of NeP across six NeP conditions: painful diabetic peripheral neuropathy, ${ }^{4}$ human immunodeficiency virus-related peripheral $\mathrm{NeP},{ }^{18}$ posttrauma/postsurgical $\mathrm{NeP}^{5}$ spinal cord injury-related NeP, ${ }^{6}$ chronic low-back pain-associated NeP, ${ }^{19}$ and smallfiber neuropathy. ${ }^{7}$ The study was approved by a central institutional review board (Concordia Clinical Research, Cedar Knolls, NJ, USA), and collection and analysis of data were compliant with the Health Insurance Portability and Accountability Act.

\section{Subjects}

Subjects with a confirmed diagnosis of any of the six conditions were identified during routine office visits at 33 community-based physician practices across the US between September 2011 and June 2012. Study sites included general practitioners $(n=9)$, neurologists $(n=7)$, pain specialists $(n=6)$, endocrinologists $(n=3)$, and other specialists (eg, orthopedist, infectious disease specialist, podiatrist, rheumatologist; $n=8$ ).

For inclusion, subjects were required to be adults ( $\geq 18$ years old) diagnosed with their NeP at least 6 months prior to enrollment, managed by the physician's practice for at least 6 months, and had experienced NeP symptoms for at least the prior 3 months. Exclusion criteria were participation in an investigational drug study in the past 6 months; presence of a serious or unstable medical or psychological condition that in the opinion of the physician would compromise participation in the study; or having a concomitant illness unrelated to $\mathrm{NeP}$ that could have confounded the assessment of NeP.

\section{Outcomes}

Subjects were asked to complete a series of self-administered, one-time questionnaires during the office visit that included patient-reported measures of pain, function, quality of life, and productivity, as previously described. ${ }^{4,6,7}$ Of relevance to the current analysis, the questionnaire included the Brief Pain Inventory - Short Form (BPI-SF) ${ }^{20}$ and the painDETECT questionnaire. ${ }^{15}$

Patients were stratified based on their level of average pain within the past 24 hours using the average pain item from the BPI-SF, which consists of an 11-point numeric rating scale $(0=$ no pain to $10=$ pain as bad as you can imagine). Painseverity levels based on previously established cut points were defined as mild (score 0-3), moderate (score 4-6), and severe (score 7-10). ${ }^{21}$

painDETECT is a nine-item questionnaire that consists of seven sensory symptom items for pain that are graded from $0=$ never to $5=$ strongly, one temporal item on pain-course pattern graded -1 to +1 , and one spatial item on pain radiation 
graded 0 for no radiation or +2 for radiating pain. A total score that ranges from -1 to 38 can be calculated from the nine items, with higher scores indicating higher levels of NeP. Although the nine-item questionnaire demonstrated strong predictive ability for NeP, principal-component analysis identified the seven sensory items as driving the data structure of the questionnaire. ${ }^{15}$ Therefore, the seven-item version of painDETECT that only includes the sensory symptom items and a total score that ranges from 0 to 35 was also evaluated for its ability to differentiate pain severity.

\section{Statistical analyses}

Scores on painDETECT were derived for subjects who had no missing responses on the sensory items (seven- and nine-item scales), and response was also required on the paincourse item on the nine-item scale. Scale-level discrimination was evaluated using unadjusted and adjusted models. ${ }^{22}$ The ability of painDETECT to discriminate between average pain-severity levels of mild, moderate, and severe, based on the average pain item from the BPI-SF as described earlier, was determined using analysis of variance or covariance models (both models used Tukey's adjustment for pair-wise comparisons) to obtain, respectively, unadjusted and adjusted mean painDETECT scores. Factors in the adjusted models were age, sex, race, ethnicity, time since NeP diagnosis, and number of comorbidities.

The corresponding mean scores on painDETECT between pairs of severity groups were translated into standardized effect sizes, ${ }^{22}$ derived by taking the difference between pair-wise means and dividing them by the pooled standard deviation on painDETECT across the three severity groups. By convention, a standardized effect size of 0.20 is considered "small", while 0.50 and 0.80 are "medium" and "large", respectively. ${ }^{23}$ Additionally, cumulative distribution of painDETECT scores by (average) pain severity were plotted and compared using the KolmogorovSmirnov test. ${ }^{24}$

Scale-level reliability was evaluated using Cronbach's alpha (covariance-based formula) ${ }^{22}$ to determine the internal consistency reliability of the nine- and seven-item versions of painDETECT. Assessment of item-level discrimination was performed using corrected item-to-total correlations ${ }^{22}$ to determine how well each item discriminated across neuropathic levels of pain as measured by painDETECT. ${ }^{22}$ These were "corrected" to account for item overlap by deleting the item under consideration from the total score. All analyses and statistics were performed using SAS version 9.2 (SAS Institute, Cary, NC, USA).

\section{Results}

\section{Subject characteristics}

The study enrolled 624 subjects who were identified with the NeP conditions, with approximately the same number of subjects from each $\mathrm{NeP}$ condition. Data on average pain severity were available for 614 subjects, with an average pain-severity distribution of $17.9 \%, 48.4 \%$, and $33.7 \%$ for mild, moderate, and severe pain, respectively (Table 1). These subjects were $55.4 \%$ male and predominantly white $(71.8 \%)$, with a mean (standard deviation) age of 55.5 (13.7) years (Table 1). However, with increasing levels of pain severity, significant trends were observed across severity levels $(P<0.05)$ toward younger age, higher proportion of females, longer duration since NeP diagnosis, and more comorbid conditions (Table 1).

Among the 614 subjects with pain-severity data, the response rate for the painDETECT questionnaire was $96.9 \%$ and $98.7 \%$ for the nine- and seven-item versions, respectively; only two subjects (one mild and one moderate pain) had nineitem scores despite a missing pain-radiation item (which was taken to indicate no radiating pain and hence given a value of 0). Similar proportions of subjects across the pain-severity categories completed both versions (Table 1).

\section{Scale-level discrimination}

On the nine-item version of painDETECT, unadjusted mean scores were 15.2, 19.8, and 24.0, for mild, moderate, and severe pain, respectively (Figure 1A). These scores differentiated between levels of average reported pain, with all pairwise comparisons statistically significant $(P<0.0001)$. The significant differentiation between all pair-wise comparisons was maintained for the adjusted mean scores of 17.3 (mild), 21.3 (moderate), and 25.3 (severe) $(P<0.0001)$ (Figure 1B). Similarly, for the seven-item version, both the unadjusted (Figure 1C) and adjusted painDETECT (Figure 1D) scores differentiated between average pain-severity levels, as indicated by the statistical significance for all pair-wise comparisons $(P<0.0001)$.

Standardized effect sizes were slightly lower (with no practical importance) for adjusted mean scores relative to unadjusted mean scores, and if anything slightly lower for the seven-item version than the nine-item version (Table 2). Both the nine-item scores and the seven-item scores showed separation with respect to the three levels of average pain severity, with strength of differentiation that was at least medium (effect sizes $\geq 0.50$ ) across all comparisons, even after adjustment (Table 2). While the magnitude of separation was in the medium range for mild versus moderate pain 
Table I Demographic and clinical characteristics by neuropathic pain severity

\begin{tabular}{|c|c|c|c|c|}
\hline Characteristic & $\operatorname{Mild}^{a}(n=\mid I 0)$ & Moderate $^{a}(n=297)$ & Severe $^{a}(n=207)$ & $P$-value ${ }^{\mathrm{b}}$ \\
\hline Age, years, mean (SD) & $58.3(15.1)$ & $55.7(13.2)$ & $53.6(13.3)$ & 0.0293 \\
\hline Sex, n (\%) & & & & 0.0222 \\
\hline Male & $71(64.5)$ & $169(56.9)$ & 101 (48.8) & \\
\hline Female & $39(35.5)$ & $128(43.1)$ & $106(5 \mid .2)$ & \\
\hline Race, n (\%) & & & & 0.0015 \\
\hline American Indian or Alaska native & I (0.9) & $3(1.0)$ & $5(2.4)$ & \\
\hline Asian & I (0.9) & $2(0.7)$ & $2(1.0)$ & \\
\hline Black or African American & $13(11.8)$ & $37(12.5)$ & $47(22.7)$ & \\
\hline White & 89 (80.9) & $230(77.4)$ & $122(58.9)$ & \\
\hline Multiracial & $2(1.8)$ & $4(1.3)$ & $5(2.4)$ & \\
\hline Other & $3(2.7)$ & $15(5.1)$ & $22(10.6)$ & \\
\hline Missing & I (0.9) & $6(2.0)$ & $4(1.9)$ & \\
\hline Ethnicity, n (\%) & & & & 0.0151 \\
\hline Non-Hispanic & $100(90.9)$ & $266(89.6)$ & $168(81.2)$ & \\
\hline Hispanic & $5(4.5)$ & $21(7.1)$ & $27(13.0)$ & \\
\hline Missing & $5(4.5)$ & $10(3.4)$ & $12(5.8)$ & \\
\hline BPI-SF pain-severity index, mean (SD) & $2.0(1.1)$ & $5.2(0.8)$ & $7.7(\mathrm{I} . \mathrm{I})$ & NA \\
\hline Time since NeP diagnosis, years, mean (SD) & $6.3(5.9)$ & $7.9(6.8)$ & $8.5(7.2)$ & 0.0059 \\
\hline Number of comorbid conditions, mean (SD) & $2.0(1.8)$ & $2.5(2.2)$ & $3.3(2.4)$ & $<0.0001$ \\
\hline Neuropathic pain condition, $\mathrm{n}(\%)$ & & & & NA \\
\hline Painful diabetic peripheral neuropathy $(\mathrm{n}=\mathrm{I} \mathrm{I} \mathrm{I})$ & $22(20.0)$ & $57(19.2)$ & $32(15.5)$ & \\
\hline $\begin{array}{l}\text { Human immunodeficiency virus-related } \\
\text { peripheral } \mathrm{NeP}(n=102)\end{array}$ & $24(21.8)$ & $4 \mid(13.8)$ & $37(17.9)$ & \\
\hline Posttrauma/postsurgical $\mathrm{NeP}(\mathrm{n}=97)$ & $14(12.7)$ & $48(16.2)$ & $35(16.9)$ & \\
\hline Spinal cord injury-related $\mathrm{NeP}(\mathrm{n}=\mathrm{I0I})$ & $21(19.1)$ & $53(17.8)$ & $27(13.0)$ & \\
\hline $\mathrm{NeP}$ in chronic low-back pain $(n=104)$ & $6(5.5)$ & $55(18.5)$ & $43(20.8)$ & \\
\hline Small-fiber neuropathy $(n=99)$ & $23(20.9)$ & $43(14.5)$ & $33(15.9)$ & \\
\hline painDETECT completion, $\mathrm{n}(\%)$ & & & & NA \\
\hline Nine-item version & $106(96.4)$ & $291(98.0)$ & $198(95.7)$ & \\
\hline Seven-item version & $108(98.2)$ & $292(98.3)$ & $206(99.5)$ & \\
\hline
\end{tabular}

Notes: aAverage pain severity was classified based on the Brief Pain Inventory - Short Form scores of 0-3 for mild, 4-6 for moderate, and 7-10 for severe pain; ${ }^{\mathrm{b} P \text {-values }}$ across pain-severity levels are from the Kruskal-Wallis test for continuous variables; from the $\chi^{2}$ test for number of comorbid conditions, and from Fisher's exact test for the remaining categorical variables.

Abbreviations: NA, not applicable; SD, standard deviation; BPI-SF, Brief Pain Inventory - Short Form; NeP, Neuropathic Pain.

and moderate versus severe pain, it was consistently large for mild versus severe pain.

The cumulative frequency-distribution plots for painDETECT scores are shown in Figure 2 for the nine-item version (Figure 2A) and the seven-item version (Figure 2B). Both versions demonstrated differentiation between painseverity levels that was statistically significant for all pair-wise comparisons $(P<0.0001)$; for each painDETECT score, the proportion of patients at that score or lower (more favorable score) was greater at lower levels of pain severity. For example, for the seven-item score, approximately $20 \%$ of subjects in the severe group had scores of 18 or lower (better), compared with approximately $45 \%$ and $70 \%$ in the moderate and mild pain groups, respectively.

\section{Scale-level reliability}

Across all subjects and pain-severity categories, corrected item-to-total correlations showed discernible and sizable amounts of item discrimination for all items on the sevenitem version, which ranged from 0.49 for "slight pressure triggers pain" to 0.62 for "light touching painful" (Table 3 ). The same set of items on the nine-item version was equally discriminating, as indicated by similar values for the corrected item-to-total correlation (Table 3). However, the two additional items showed low or no discrimination (pain radiation, 0.21; pain course, -0.08). Despite having two fewer items, the seven-item version gave a slightly higher Cronbach's alpha (0.80) than the nine-item version (0.76).

\section{Discussion}

This study compellingly demonstrated the psychometric validity and reliability of painDETECT for distinguishing mild, moderate, and severe levels of average pain in subjects with NeP, with pain severity rated using the average pain item from the BPI-SF. Specifically developed screening instruments, such as painDETECT, have been shown to be 
A

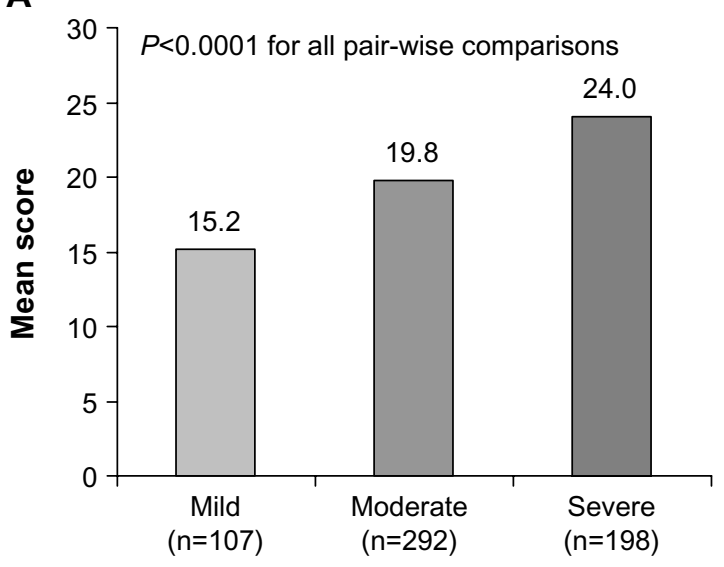

C

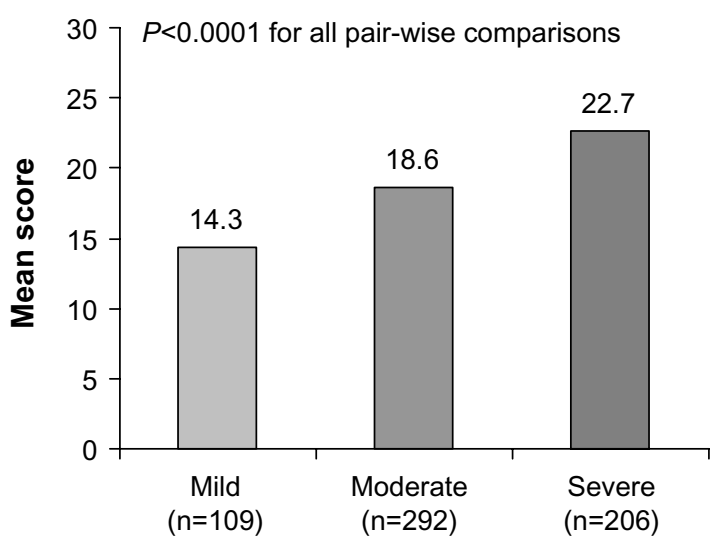

B

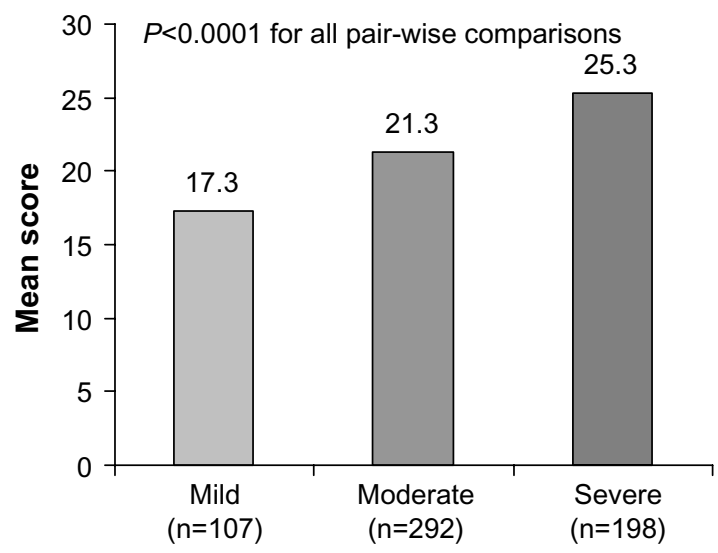

D

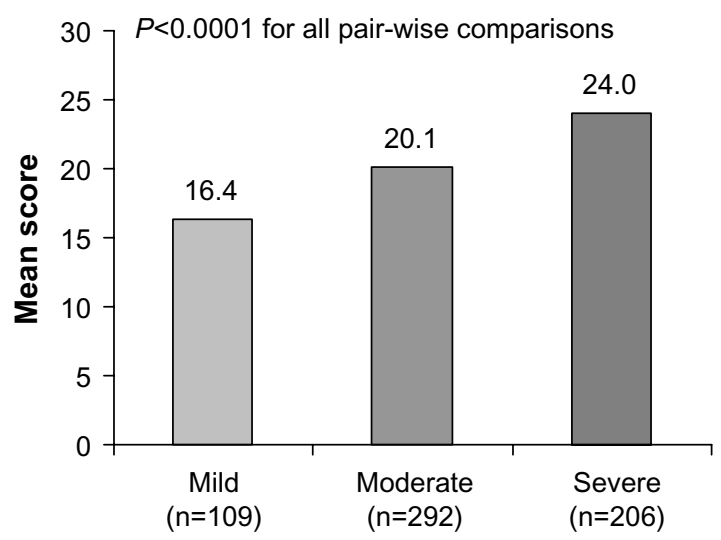

Figure I (A-D) Mean painDETECT scores across pain-severity categories for average pain. (A) Nine-item unadjusted scores; (B) nine-item adjusted scores; (C) seven-item unadjusted scores; (D) seven-item adjusted scores.

useful in identifying the likelihood that pain is of neuropathic origin. ${ }^{11}$ Although there is a need for characterizing and quantifying NeP concurrently with its identification in patients with various $\mathrm{NeP}$ conditions, to our knowledge, no other NeP instrument has yet been specifically validated for distinguishing between levels of average pain severity. These results therefore complement and extend studies that have

Table 2 Standardized effect sizes for pair-wise comparisons of painDETECT scores

\begin{tabular}{|c|c|c|c|c|}
\hline \multirow{3}{*}{$\begin{array}{l}\text { Comparison } \\
\text { between } \\
\text { average pain- } \\
\text { severity levels }\end{array}$} & \multicolumn{4}{|c|}{ Standardized effect size $^{a}$} \\
\hline & \multicolumn{2}{|c|}{ Nine-item version } & \multicolumn{2}{|c|}{ Seven-item version } \\
\hline & Unadjusted & Adjusted & Unadjusted & Adjusted \\
\hline $\begin{array}{l}\text { Mild versus } \\
\text { moderate }\end{array}$ & -0.65 & -0.56 & -0.63 & -0.55 \\
\hline $\begin{array}{l}\text { Mild versus } \\
\text { severe }\end{array}$ & -1.25 & -1.13 & -1.23 & -1.12 \\
\hline $\begin{array}{l}\text { Moderate } \\
\text { versus severe }\end{array}$ & -0.60 & -0.57 & -0.60 & -0.57 \\
\hline
\end{tabular}

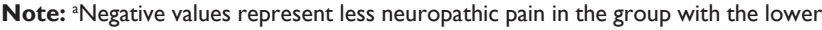
level of average pain severity. suggested that NeP-screening instruments may help distinguish among sensory symptom profiles and pain-severity levels. ${ }^{16,17,25-27}$

Both the seven-item and nine-item scores of painDETECT provided good discrimination (known-group validity) of average pain severity, with significant differentiation $(P<0.0001)$ for all pair-wise comparisons between mild, moderate, and severe pain. The extent of the separation was substantial based on estimation of standardized effect sizes: medium magnitude for mild versus moderate pain and for moderate versus severe pain, and large magnitude for mild versus severe pain. Although it can be expected that a similar set of results would apply to the similarly phrased BPI-SF questions on worst pain, least pain, and current pain, further research would be needed for their confirmation.

These findings, while new and clinically relevant, are not entirely unexpected, given the previously observed relationship between painDETECT and pain severity. The initial painDETECT-validation study reported a significant association $(P<0.001)$ between pain severity (average over 


\section{A}

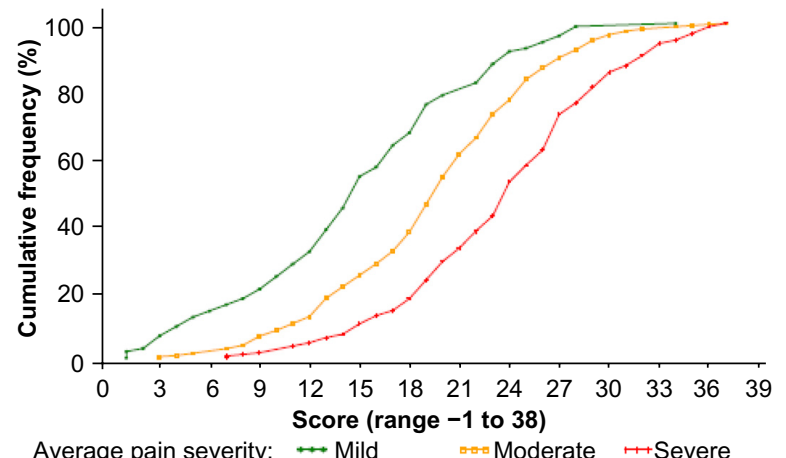

B

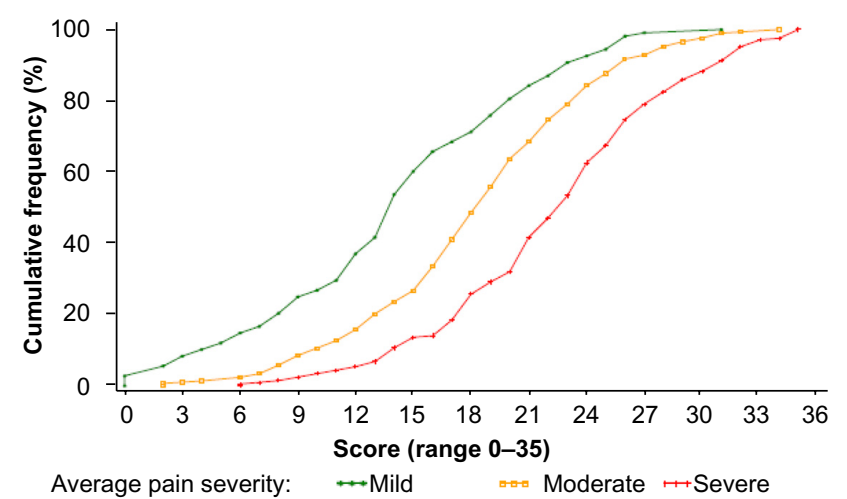

Average pain severity: $\quad$ Mild Moderate $\rightarrow$ Severe

Figure 2 Cumulative frequency distribution by average pain severity for (A) nineitem painDETECT scores and (B) seven-item painDETECT scores.

the past 4 weeks) and painDETECT scores, ${ }^{15}$ and other studies have confirmed that higher painDETECT scores were significantly associated with greater average pain severity over the previous 4 weeks $(P<0.05){ }^{16,17}$ However, those studies evaluated the relationship within the framework of the painDETECT cutoff values for differentiating NeP from nociceptive pain. In contrast, the current study started with a large and varied $\mathrm{NeP}$ population, and quantified both unadjusted and adjusted mean painDETECT values based on pain-severity levels.

Table 3 Corrected item-to-total correlations for the nine- and seven-item painDETECT

\begin{tabular}{lll}
\hline painDETECT item & $\begin{array}{l}\text { Nine-item } \\
\text { version }\end{array}$ & $\begin{array}{l}\text { Seven-item } \\
\text { version }\end{array}$ \\
\hline Burning sensation & 0.48 & $0.5 \mathrm{I}$ \\
Tingle or prickling sensation & 0.59 & $0.6 \mathrm{I}$ \\
Light touching painful & 0.60 & 0.62 \\
Sudden pain attacks like electric shocks & 0.49 & 0.50 \\
Cold or heat painful & 0.55 & 0.54 \\
Sensation of numbness & 0.45 & 0.48 \\
Slight pressure triggers pain & 0.49 & 0.49 \\
Pain radiation & 0.21 & - \\
Pain course & -0.08 & - \\
\hline
\end{tabular}

Two previous studies have also suggested that painsymptom descriptors may be significant predictors of NeP severity. ${ }^{28,29}$ In those studies, the presence of a higher number of descriptors, as well as specific descriptor qualities, had greater predictive value for more severe pain. ${ }^{28,29}$ The range of descriptors constituting painDETECT overlaps with those descriptors that have shown predictive ability for pain severity. Taken in context, the current results confirm and supplement the relevance of using descriptors for characterizing NeP, including for quantifying severity, and provide reassuring evidence that the observed results enhance the clinical usefulness of painDETECT in ways not previously validated.

Both the seven-item version and the nine-item version demonstrated internal consistency reliability that was acceptable (above 0.70). However, the seven-item version resulted in a slightly higher reliability (Cronbach's $\alpha=0.80$ ) than the nine-item version (Cronbach's $\alpha=0.76$ ) and more consistent item-level discrimination. The reliability of the seven-item version is not only similar to that reported in the validation study (Cronbach's $\alpha=0.83$ ), ${ }^{15}$ but its higher value than the nine-item version is also in concordance with previous observations, ${ }^{27}$ suggesting that the two nonsensory items are not psychometrically compatible with the sensory items. While further confirmation for the greater reliability of the seven-item version is needed, it can nevertheless be recommended that the seven sensory items be used together; the two nonsensory items, on temporal and spatial characterization of the pain pattern, that are included in the nine-item version can be analyzed and interpreted separately.

It should also be noted that since the population in the current study consisted of patients with confirmed NeP, the cumulative distribution curves are in marked contrast to those in studies evaluating discrimination between main pain subtypes (nociceptive, pure neuropathic, mixed) where the nociceptive group clearly resulted in lower (more favorable) scores on painDETECT. ${ }^{15,27}$ In addition, in the current study, the cumulative distributions convincingly showed that painDETECT scores were dependent on the degree of average pain severity for patients with confirmed NeP; fitting only one curve for all patients with $\mathrm{NeP}$ (ie, without regard to average pain severity), as has been done previously, ${ }^{15,27}$ would mask this finding.

At least two possible limitations of this research should be noted. One is that the study was cross-sectional rather than longitudinal. The opportunity to link changes in painDETECT scores to changes in average pain severity was not available. However, painDETECT has generally been used 
as a screening tool at a single time point, since its responsiveness to change over time or with treatment has not been adequately evaluated.

The second limitation is that this study could be potentially criticized for evaluation of a population that included different NeP conditions, since it can be argued that there may be differences among the conditions resulting from their various etiologies. However, it has been reported that there appear to be greater similarities than differences overall with regard to NeP regardless of etiology. ${ }^{25,26}$

Furthermore, a recent study suggested that there may be up to four distinct pain-characteristic profiles that are independent of etiology. ${ }^{26}$ Therefore, since the scoring of painDETECT is based on an amalgam of frequency and severity for relevant individual sensory symptoms, severity discrimination will likely be maintained even across the different sensory symptom profiles, supporting a robust ability of painDETECT to both identify $\mathrm{NeP}$ and profile its sensitivity with respect to average pain regardless of the etiology or sensory profile. It could be argued that the wide range of average pain severity and $\mathrm{NeP}$ across etiologies is intended to foster a diverse and expansive base of patients that resounds in drawing stronger psychometric results and inferences to patients with $\mathrm{NeP}$ regardless of etiology.

\section{Conclusion}

This study provides strong psychometric evidence on the validity of painDETECT for distinguishing scores on $\mathrm{NeP}$ based on scores of average pain severity for patients with NeP. Therefore, the ability of painDETECT to discriminate among mild, moderate, and severe levels of average pain further supports the measurement properties and clinical relevance of this instrument for identifying and characterizing NeP. The nine-item and seven-item versions of painDETECT also show satisfactory reliability, but the higher reliability of the seven-item version suggests it may be more prudent to use this version, with the two nonsensory items used separately to further characterize NeP. Additional investigation of the psychometric properties of painDETECT and other $\mathrm{NeP}$-screening instruments are warranted, since more comprehensive characterization of $\mathrm{NeP}$ at screening can inform management strategies that may help improve outcomes and reduce the disease burden.

\section{Disclosure}

This study was funded by Pfizer. Joseph C Cappelleri and Alesia Sadosky are employees and shareholders of Pfizer, the sponsor of this study; Vijaya Koduru is an employee of the
Eliassen Group, who were paid consultants to Pfizer in connection with the analysis described in this study; E Jay Bienen is an independent scientific consultant who was funded by Pfizer in connection with manuscript development.

\section{References}

1. International Association for the Study of Pain. IASP taxonomy. 2012. Available from: https://www.iasp-pain.org/Education/Content. aspx?ItemNumber=1698. Accessed April 5, 2014.

2. van Hecke O, Austin SK, Khan RA, Smith BH, Torrance N. Neuropathic pain in the general population: a systematic review of epidemiological studies. Pain. 2014;155(4):654-662.

3. Taylor-Stokes G, Pike J, Sadosky A, Chandran A, Toelle T. Association of patient-rated severity with other outcomes in patients with painful diabetic peripheral neuropathy. Diabetes Metab Syndr Obes. 2011;4: 401-408.

4. Sadosky A, Schaefer C, Mann R, et al. Burden of illness associated with painful diabetic peripheral neuropathy among adults seeking treatment in the US: results from a retrospective chart review and cross-sectional survey. Diabetes Metab Syndr Obes. 2013;6:79-92.

5. Parsons B, Schaefer C, Mann R, et al. Economic and humanistic burden of post-trauma and post-surgical neuropathic pain among adults in the United States. J Pain Res. 2013;6:459-469.

6. Mann R, Schaefer C, Sadosky A, et al. Burden of spinal cord injuryrelated neuropathic pain in the United States: retrospective chart review and cross-sectional survey. Spinal Cord. 2013;51(7):564-570.

7. Schaefer C, Mann R, Sadosky A, et al. Health status, function, productivity, and costs among individuals with idiopathic painful peripheral neuropathy with small fiber involvement in the United States: results from a retrospective chart review and cross-sectional survey. $\mathrm{J} \mathrm{Med}$ Econ. 2014;17(6):394-407.

8. Dworkin RH, O'Connor AB, Kent J, et al. Interventional management of neuropathic pain: NeuPSIG recommendations. Pain. 2013;154(11): 2249-2261.

9. Dworkin RH, O'Connor AB, Audette J, et al. Recommendations for the pharmacological management of neuropathic pain: an overview and literature update. Mayo Clin Proc. 2010;85(Suppl 3):S3-S14.

10. Attal N, Cruccu G, Baron R, et al. EFNS guidelines on the pharmacological treatment of neuropathic pain: 2010 revision. Eur J Neurol. 2010;17(9):1113-1123.

11. Bennett MI, Attal N, Backonja MM, et al. Using screening tools to identify neuropathic pain. Pain. 2007;127(3):199-203.

12. Bouhassira D, Attal N, Alchaar H, et al. Comparison of pain syndromes associated with nervous or somatic lesions and development of a new neuropathic pain diagnostic questionnaire (DN4). Pain. 2005;114(1-2): 29-36.

13. Bennett M. The LANSS Pain Scale: the Leeds assessment of neuropathic symptoms and signs. Pain. 2001;92(1-2):147-157.

14. Bennett MI, Smith BH, Torrance N, Potter J. The S-LANSS Score for identifying pain of predominantly neuropathic origin: validation for use in clinical and postal research. J Pain. 2005;6(3):149-158.

15. Freynhagen R, Baron R, Gockel U, Tölle TR. painDETECT: a new screening questionnaire to identify neuropathic components in patients with back pain. Curr Med Res Opin. 2006;22(10):1911-1920.

16. Ohtori S, Orita S, Yamashita M, et al. Existence of a neuropathic pain component in patients with osteoarthritis of the knee. Yonsei Med J. 2012;53(4):801-805.

17. Elias LA, Yilmaz Z, Smith JG, et al. PainDETECT: a suitable screening tool for neuropathic pain in patients with painful post-traumatic trigeminal nerve injuries? Int J Oral Maxillofac Surg. 2014;43(1):120-126.

18. Mann R, Bergstrom F, Schaefer C, et al. Characteristics of subjects with human immunodeficiency virus-related neuropathic pain in the United States: BEAT Neuropathic Pain Observational Study. Poster presented at: American Society of Regional Anesthesia and Pain Medicine 11th Annual Pain Medicine Meeting; November 15-18, 2012; Miami, FL. 
19. Sadosky A, Schaefer C, Parsons B, et al. Characteristics of subjects with chronic low back pain-related neuropathic pain (CLBP-NeP) in the US: BEAT Neuropathic pain observational study [abstract]. Ann Neurol. 2012;72 Suppl:117.

20. Cleeland CS, Ryan KM. Pain assessment: global use of the Brief Pain Inventory. Ann Acad Med Singapore. 1994;23(2):129-138.

21. Zelman D, Dukes E, Brandenburg N, Bostrom A, Gore M. Identification of cut-points for mild, moderate and severe pain due to diabetic peripheral neuropathy. Pain. 2005;115(2):29-36.

22. Cappelleri JC, Zou KH, Bushmakin AG, Alvir JM, Alemayehu D, Symonds T. Patient-Reported Outcomes: Measurement, Implementation and Interpretation. Boca Raton (FL): Chapman and Hall/CRC; 2014.

23. Cohen J. Statistical Power Analysis for the Behavioral Sciences. 2nd ed. Hillsdale (NJ): Lawrence Erlbaum Associates; 1988.

24. Gibbons JC, Chakraborti S. Nonparametric Statistical Inference. 5 th ed. Boca Raton (FL): Chapman and Hall/CRC; 2011.
25. Baron R, Forster M, Binder A. Subgrouping of patients with neuropathic pain according to pain-related sensory abnormalities: a first step to a stratified treatment approach. Lancet Neurol. 2012;11(11):999-1005.

26. Freeman R, Baron R, Bouhassira D, Cabrera J, Emir B. Sensory profiles of patients with neuropathic pain based on the neuropathic pain symptoms and signs. Pain. 2014;155(2):367-376.

27. De Andrés J, Pérez-Cajaraville J, Lopez-Alarcón MD, et al. Cultural adaptation and validation of the painDETECT scale into Spanish. Clin J Pain. 2012;28(3):243-253.

28. Backonja MM, Stacey B. Neuropathic pain symptoms relative to overall pain rating. J Pain. 2004;5(9):491-497.

29. Butler S, Jonzon B, Branting-Ekenback C, Wadell C, Farahmand B. Predictors of severe pain in a cohort of 5271 individuals with selfreported neuropathic pain. Pain. 2013;154(1):141-146.

\section{Publish your work in this journal}

ClinicoEconomics \& Outcomes Research is an international, peerreviewed open-access journal focusing on Health Technology Assessment, Pharmacoeconomics and Outcomes Research in the areas of diagnosis, medical devices, and clinical, surgical and pharmacological intervention. The economic impact of health policy and health systems organization also constitute important areas of coverage. The manuscript management system is completely online and includes a very quick and fair peer-review system, which is all easy to use. Visit http://www.dovepress.com/testimonials.php to read real quotes from published authors.

Submit your manuscript here: http://www.dovepress.com/clinicoeconomics-and-outcomes-research-journal 